\title{
Cigarette smoking, nicotine dependence and anxiety disorders: a systematic review of population-based, epidemiological studies
}

\author{
Steven Moylan ${ }^{1 *}$, Felice N Jacka ${ }^{1,2}$, Julie A Pasco ${ }^{1,3}$ and Michael Berk ${ }^{1,2,4,5}$
}

\begin{abstract}
Background: Multiple studies have demonstrated that rates of smoking and nicotine dependence are increased in individuals with anxiety disorders. However, significant variability exists in the epidemiological literature exploring this relationship, including study design (cross-sectional versus prospective), the population assessed (random sample versus clinical population) and diagnostic instrument utilized.

Methods: We undertook a systematic review of population-based observational studies that utilized recognized structured clinical diagnostic criteria (Diagnostic and Statistical Manual of Mental Disorders (DSM) or International Classification of Diseases (ICD)) for anxiety disorder diagnosis to investigate the relationship between cigarette smoking, nicotine dependence and anxiety disorders.
\end{abstract}

Results: In total, 47 studies met the predefined inclusion criteria, with 12 studies providing prospective information and 5 studies providing quasiprospective information. The available evidence suggests that some baseline anxiety disorders are a risk factor for initiation of smoking and nicotine dependence, although the evidence is heterogeneous and many studies did not control for the effect of comorbid substance use disorders. The identified evidence however appeared to more consistently support cigarette smoking and nicotine dependence as being a risk factor for development of some anxiety disorders (for example, panic disorder, generalized anxiety disorder), although these findings were not replicated in all studies. A number of inconsistencies in the literature were identified.

Conclusions: Although many studies have demonstrated increased rates of smoking and nicotine dependence in individuals with anxiety disorders, there is a limited and heterogeneous literature that has prospectively examined this relationship in population studies using validated diagnostic criteria. The most consistent evidence supports smoking and nicotine dependence as increasing the risk of panic disorder and generalized anxiety disorder. The literature assessing anxiety disorders increasing smoking and nicotine dependence is inconsistent. Potential issues with the current literature are discussed and directions for future research are suggested.

Keywords: agoraphobia, anxiety disorder, cigarette smoking, epidemiology, generalized anxiety disorder, nicotine dependence, obsessive-compulsive disorder, panic disorder, post-traumatic stress disorder, specific phobia

\section{Background}

Anxiety disorders (ADs) represent the most common mental illness diagnoses across many countries [1,2] and are associated with significant impairment to health and quality of life [3,4]. Multiple population-based epidemiological studies have identified increased rates of smoking

\footnotetext{
* Correspondence: steven.moylan@deakin.edu.au

'Deakin University School of Medicine, Barwon Health, Geelong, Victoria, Australia

Full list of author information is available at the end of the article
}

amongst individuals with mental illness, and increased rates of mental illness amongst smokers [5]. Specifically, ADs have been reported to be associated with increased rates of smoking, increased consumption of cigarettes per smoker, and lower rates of smoking cessation than non-anxiety disordered control groups [6,7]. In addition, increased anxiety symptoms as measured on symptom scales appear to be correlated with increased rates of smoking [8].

\section{Biomed Central}

(c) 2012 Moylan et al; licensee BioMed Central Ltd. This is an Open Access article distributed under the terms of the Creative Commons Attribution License (http://creativecommons.org/licenses/by/2.0), which permits unrestricted use, distribution, and reproduction in any medium, provided the original work is properly cited. 
The relationship between ADs, smoking behavior and nicotine dependence (ND) could be explained by three non-mutually exclusive relationships. Firstly, smoking behavior and/or ND may increase the chances of developing an AD. Potential mechanisms underpinning this include adverse effects of smoking on neurodevelopment and neurotransmitter pathways modulating anxiety that may predispose individuals to developing enhanced anxiety, or direct effects to the respiratory and autonomic systems that may alter physical responses to anxiety provoking situations $[9,10]$. Secondly, ADs may increase smoking behavior and the risk for ND. Mechanisms for this could include a propensity for those with increased anxiety to commence smoking, or use of cigarettes as an anxiolytic self-treatment $[11,12]$. Thirdly, the relationship may be underpinned by a shared vulnerability factor or group of factors that increase the likelihood of smoking, ND and AD development (for example, low socioeconomic status) [2,13].

However, a number of inconsistencies exist within the current literature examining the relationships between cigarette smoking, ND and ADs. Firstly, many currently available population-based epidemiological investigations have utilized clinical symptom severity scales rather than validated diagnostic instruments to make AD diagnoses. Secondly, many studies utilizing validated diagnostic instruments have been cross-sectional, and therefore unable to provide insight into the direction of causality underpinning the association. Thirdly, many investigations have been drawn from clinical populations as opposed to whole population samples, potentially introducing selection biases. An accurate assessment of the association between cigarette smoking, ND and validated ADs, including direction of causality and potential variation amongst differing anxiety disorder subgroups or populations, may help inform targeted interventions in at risk populations.

This paper aims to critically review the available epidemiological studies that have utilized general population samples and validated diagnostic instruments based upon recognized Diagnostic and Statistical Manual (DSM) [14] or International Classification of Diseases (ICD) systems [15], exploring the relationship between cigarette smoking, ND and ADs. Structured diagnostic instruments have advantages, as they are replicable across studies, and in some cases include an interview (for example, the Composite International Diagnostic Interview (CIDI)). These interviews often document date of onset and recent symptom expression, allowing for assessments of temporality. A particular focus for this review is on studies that have utilized prospective designs and can therefore provide insight into the direction of the potential underlying causal relationships.

\section{Methods}

\section{Systematic review search strategy}

A systematic review of the English language literature exploring relationships between ADs, ND and cigarette smoking was undertaken. The aim was to provide a descriptive overview of the available literature, with a focus on information that could inform understanding of direction of causality. A computerized search strategy of medical databases PubMed and EMBASE utilized the following search strategies, was limited to English language literature and human studies. No date restrictions were placed. PubMed: 'Anxiety Disorders' (MeSH) AND (Smoking(Title/Abstract) OR Tobacco(Title/Abstract) OR Nicotine(Title/Abstract) OR Cigarette(Title/ Abstract)) AND Anxiety AND ('humans' (MeSH terms) AND English); EMBASE: ‘\#1 AND \#2 AND \#3' where $\# 1$ = "anxiety disorder'/exp AND (humans)/lim AND (english)/lim AND (embase)/lim', \#2 = 'smoking:ab OR tobacco:ab OR cigarette:ab OR nicotine:ab AND (humans)/lim AND (english)/lim AND (embase)/lim' and \#3 = "anxiety'/exp AND (humans)/lim AND (english)/lim AND (embase)/lim'.

\section{Extraction of references}

All extracted references were combined and duplicate references deleted. Titles and abstracts of all references were initially assessed for relevance to the review topic. Full texts of references identified as potentially containing relevant information were assessed against the predetermined inclusion criteria for quality and relevance. For completeness, bibliographies of extracted references were manually searched for further relevant references. Where relevant references were discovered, their bibliographies were manually searched.

\section{Inclusion criteria}

The following inclusion criteria were applied to the available references. Included studies needed to utilize a random sample, drawn from the general population, investigating the association between ADs, ND and cigarette smoking. The included population must have been assessed for panic disorder (PD), generalized anxiety disorder (GAD), obsessive-compulsive disorder (OCD), social phobia (SP), specific phobia (SPP), post-traumaticstress disorder (PTSD) and/or agoraphobia (AG), utilizing a recognized and validated structured diagnostic tool or documented clinician diagnosis in line with either ICD-9/ICD-10 or DSM-III or DSM-IV criteria. The criteria establishing cigarette smoking must have been clearly documented, either as dichotomous (yes/no) or through frequency of cigarette consumption. ND must have been diagnosed through a recognized diagnostic tool linked to ICD or DSM criteria. The criteria for ND 
mirror those of other substance dependence disorders, and include (amongst others) symptoms of tolerance, withdrawal, increasing use and difficulty quitting. All data must have represented new information and not replication of previous study results.

\section{Literature search and application of inclusion criteria}

The structured computerized literature search was performed on 18 November 2011 yielded 298 references from PubMed and 138 references from EMBASE. In all, 36 records were duplicated after compilation of search results, leaving 400 unique records. Initial review of titles and abstracts revealed 91 records with potentially relevant information for the review. Manual searching of the bibliographies from these 91 references revealed 16 further potential references. Full text review of these 107 references revealed 47 studies meeting the inclusion criteria. See Figure 1 for search strategy flowchart and Figure 2 for breakdown of studies.

\section{Data extraction}

All data were extracted upon the following variables: (1) study name, (2) study population, (3) study design (prospective, quasiprospective or cross-sectional), (4) definition of AD diagnosis, (5) definition of smoking status, (6) statistical parameters utilized and (7) primary results presented. A focus on measures of association (for example, odds ratio (OR), hazard ratio (HR)) was taken. Where available, both unadjusted and adjusted measures were extracted. Data were extracted into a preprepared structured Microsoft Excel database (Microsoft; Redmond, WA, USA).

\section{Results}

There were 47 studies meeting inclusion criteria. The studies were broken down by type and direction of analysis (prospective, quasiprospective and cross-sectional) and are represented in Additional files 1, 2, 3, 4, 5.

\section{Prospective studies}

A total of 13 studies were identified that utilized population-based samples to assess a prospective relationship between ADs, smoking behavior, and ND. These studies comprised random population samples drawn from the United States, New Zealand, Germany and The Netherlands, with some samples used in multiple studies. Discussion of the included studies has been grouped below based on the sample used.

\section{Influence of anxiety disorders on risk of later smoking or nicotine dependence}

(A summary of this section is as follows (see also Additional file 1, Table S1): the best available evidence is equivocal, but suggests that certain baseline ADs are risk factors for onset of smoking and nicotine dependence, although results vary across studies and across different disorders. The best available studies failed to control for comorbid substance use disorders.)

The first published prospective data were drawn from the Oregon Adolescent Project Depression project, which randomly recruited adolescents (aged 14 years to 18 years) from high schools in Western Oregon, USA in 1987 to 1989 and followed them at two time points (1 year later, and on their 24th birthday). Assessment of ADs was undertaken utilizing the Schedule for Affective Disorders and Schizophrenia for School-Age Children (K-SADS) for DSM-III revision (DSM-III-R) [16], and smokers were defined as those smoking $\geq 3$ times per week. Brown et al. [17] demonstrated no difference in the odds for incident smoking at 1-year follow-up in those with versus without any ADs at baseline, both unadjusted and after controlling for a variety of demographic and other risk factors. This finding of no association was replicated in the 24-year-old follow-up [18] although only PD, and not grouped ADs, was used as the exposure variable.

Breslau et al. [19] utilized a random population sample of 1,007 young adults (21 years to 30 years), drawn from the Detroit Epidemiologic Study, to examine PD as a predictor of cigarette smoking. Baseline assessment occurred in 1989 and three follow-ups (1990, 1992 and 1994) were conducted. Diagnosis of PD was made through use of the National Institute of Mental Health Diagnostic Interview Schedule [20] for DSM-III-R criteria, and 'daily smoking' was defined as smoking daily for $\geq 1$ month. Risk of smoking onset was increased in those with PD at baseline (HR $=2.20$ (95\% CI 1.10 to 4.42$)$ ), but this significance was lost when controlled for the presence of major depressive disorder (MDD). Breslau et al. [21] extended their investigation, incorporating two further follow-ups (1999 and 2001) to assess the interaction between PTSD and ND over 10 years. After adjusting for gender, race and education, the odds of incident ND at follow-up was 4.03 (95\% CI 2.10 to 7.72 ) in those with baseline PTSD versus those without a trauma history.

Johnson et al. [22] followed a random sample of adolescents from New York state, assessed at baseline in 1983 (mean age 14 years) and at two subsequent follow-ups in 1985 to 1986 (mean age 16 years) and 1991 to 1993 (mean age 22 years). Diagnosis of ADs (grouped and individual) at baseline was made using the Diagnostic Interview Schedule for Children (DIS-C) [23] to DSM-III criteria. Smoking assessment was by self-report, and categorized into smoking $>1$ pack $(20+$ cigarettes $)$ versus smoking $<1$ pack (1 to 19 cigarettes) per day (not non-smokers). After adjusting for a variety of demographic and other risk factors no association was detected between adolescent anxiety disorder status and onset of smoking in adulthood.

The Early Developmental Stages of Psychopathology (EDSP) study has been utilized to assess the association 


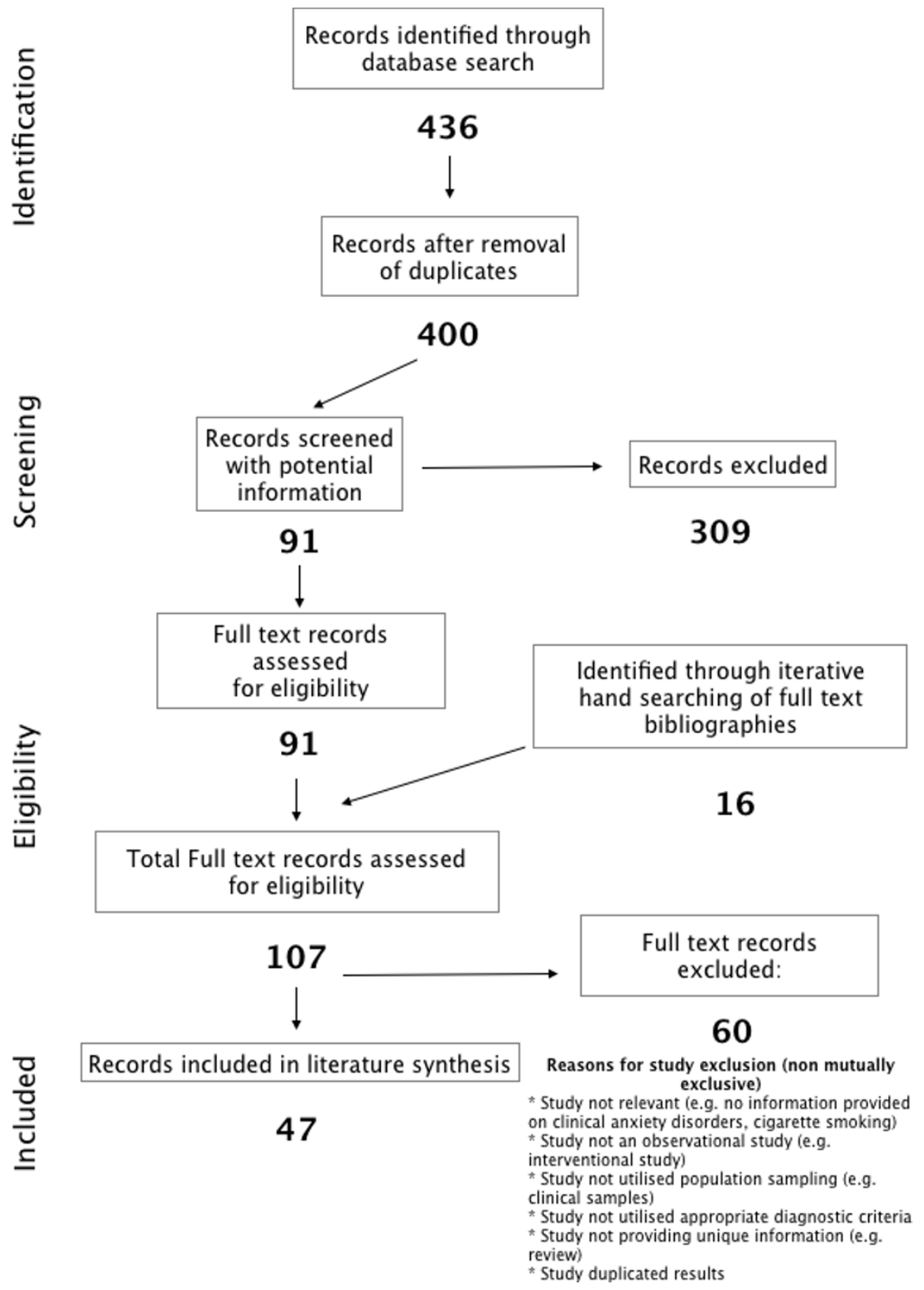

Figure 1 Preferred Reporting Items for Systematic Reviews and Meta-Analyses (PRISMA) search strategy flow diagram.

between ADs, ND and smoking behavior. In this study, a random community cohort of 3,021 adolescents and young adults (age 14 years to 24 years) was sampled from metropolitan Munich. The cohort was assessed at baseline (1995) and two follow-ups, the first between 1996 and 1997 and the second between 1998 and 1999 (a third follow-up was subsequently conducted in 2005 to 2006) [24]. $\mathrm{AD}$ and ND diagnoses were made utilizing an updated version [25] of the Composite International Diagnostic
Interview [26] for DSM-IV criteria and smoking was assessed by self-report. Isensee et al. [27] categorized participants from the EDSP into non-smokers, occasional smokers, non-dependent regular smokers and dependent regular smokers (see Additional file 1, Table S1 for definitions) and calculated odds ratios for incident smoking by baseline AD status. No associations were found between baseline AD status and odds of incident smoking. Sonntag et al. [28] extended this study in SP but once again found 


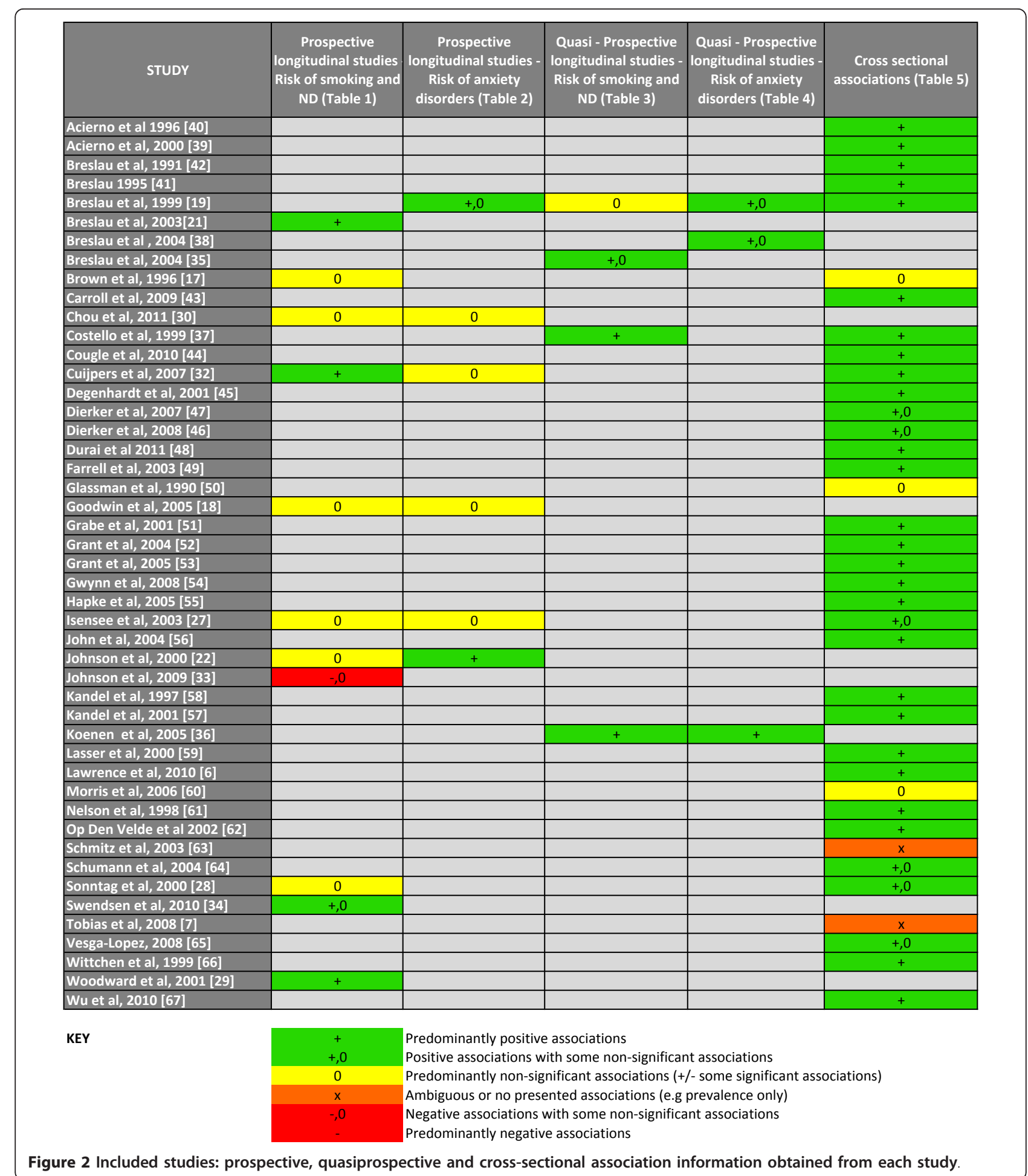

no association, although a positive association was found between those with social fears symptoms and later development of ND.

Woodward et al. [29] utilized a New Zealand birth cohort to compare the risk of DSM-IV ND between the ages of 18 and 21, dependent upon diagnosis of ADs between the ages of 14 to 16 . A linear association was found between increasing number of ADs at age 14 to 16 (0 to $3+$ ) and subsequent ND diagnosis, although this was not significant after controlling for childhood sexual abuse, alcohol abuse, parental changes and deviant peer affiliations. 
Chou et al. [30] utilized the National Epidemiologic Survey on Alcohol and Related Conditions (NESARC) to investigate the association between ADs and ND in adults age 60 years and older $(n=8,012)$. Diagnoses were made utilizing the Alcohol Use Disorders and Associated Disabilities Interview Schedule for DSM-IV criteria (AUDADIS-IV) [31] and the risk of incident ND was assessed between baseline (2000 to 2001) and follow-up (2004 to 2005). No associations were found between baseline AD status and subsequent ND.

Cuijpers et al. [32] utilized data from the Netherlands Mental Health Survey and Incident Study (NEMESIS) to investigate the relationship between incident ADs (expressed as incident rate ratios) and previous smoking status. The NEMESIS study randomly recruited adults (18 years to 64 years) from 90 municipalities in The Netherlands, undertaking baseline assessments and 2 follow-ups at 1 year and 3 years $(n=4,796)$. The CIDI [26] was used for DSM-III-R diagnoses, and smoking was assessed by self-report, with participants placed into 4 categories (non-smokers, 1 to 9 cigarettes daily, 10 to 19 cigarettes daily and $20+$ cigarettes daily). In a follow-up analysis, having 12 -month (incidence rate ratio (IRR) $4.46(P<0.05))$ and lifetime GAD (IRR $4.46(P<0.05))$ was associated with increased risk of smoking onset at follow-up.

Johnson et al. [33] utilized the prospective follow-up of the full NESARC database $(n=34,653)$ to assess the impact of $\mathrm{AD}$ on smoking onset and persistence. Grouped ADs were associated with reduced daily smoking onset (OR 0.62 (95\% CI 0.39 to 0.99$)$ ) when adjusting for demographics and socioeconomic status, but not with smoking persistence. Interestingly, comorbid substance use was an effect modifier; respondents with a comorbid substance use disorder (for example, alcohol, marijuana, amphetamines, opioids, sedatives, tranquilizers, cocaine, inhalants, hallucinogens, heroin, and other drugs) and AD demonstrated an increased risk of daily smoking onset (OR 2.22 (1.01 to 4.91)), whereas those without comorbid substance use disorder had a decreased risk of smoking onset (OR 0.43 (0.23 to 0.78)).

Most recently, Swendsen et al. [34] utilized a 10-year follow-up of 5,001 participants drawn from the National Co-morbidity Survey (NCS) to investigate mental disorders as a risk factor for onset of daily smoking or ND. The NCS, conducted in the US between 1990 and 1992, was a stratified multistage probability sample of 8,098 noninstitutionalized residents (age 15 years to 54 years), utilizing the CIDI (V1.1), that assessed the interaction between smoking, ND and DSM-III-R mental disorders. The NCS2, performed in 2001 to 2002, was a 10-year re-interview of 5,001 participants from the NCS, but which utilized an updated version CIDI (V3.0) for DSM-IV criteria. After adjusting for sociodemographic characteristics, the odds of commencing daily smoking was increased for respondents with baseline PD, SP, GAD and specific phobia, but not for those with baseline PTSD or agoraphobia. In contrast, in those respondents with baseline daily smoking the odds of ND onset were raised in PTSD, agoraphobia and specific phobia, but not PD, SP or GAD. When considering the whole population odds of ND onset, having baseline PTSD, SP and specific phobia conferred an increased risk (see Additional file 1, Table S1).

\section{Smoking and nicotine dependence as risk factors for later anxiety disorders}

(A summary of this section is as follows (see also Additional file 2, Table $\mathrm{S} 2$ ): the available prospective evidence associating smoking and nicotine dependence as risk factors for incident anxiety disorders is limited and heterogeneous. However, smoking has been demonstrated as a risk factor for grouped anxiety disorders, panic disorder and generalized anxiety disorder in a number of studies, although these findings are not replicated in all studies.)

Data from the Oregon Adolescent Depression Project were utilized to assess the relationship between baseline smoking status and incident ADs. Goodwin et al. [18] demonstrated an association between increased odds of PD diagnosis at age 24 in those with daily smoking at baseline versus those not smoking daily (OR 5.1 (2.4 to 10.5)), which remained significant after controlling for other ADs and parental risk factors. No other associations were found.

Utilizing data from the Detroit Epidemiologic Study, Breslau et al. [19] found increased risk of subsequent PD onset in individuals with prior daily smoking even when controlling for gender and MDD (HR 13.13 (4.41 to 39.10)). In addition, prior daily smokers who continued to smoke were more likely to experience incident PD (HR 14.46 (4.81 to 43.5$)$ ) when controlled for gender and MDD.

In the New York Adolescent Cohort, relationships were discovered between odds of adult ADs when grouped (OR 10.78 (1.48 to 78.55)), GAD (OR 5.53 (1.84 to 16.66) and PD (OR 15.58 (2.31 to 105.14) when comparing baseline $>1$ pack per day smokers versus $<1$ pack per day smokers [22]. Data from the EDSP studies [27] demonstrated relationships between increased incident PD, agoraphobia, SP and PTSD when comparing baseline ND smokers versus non-users, however all associations became non-significant when controlled for comorbid conditions at baseline (depressive disorders, panic attacks, other ADs, alcohol and drug disorders, and eating disorders). In the NESARC study, Chou et al. [30] assessed the relationship between ND at baseline and subsequent $\mathrm{ADs}$, finding no associations.

Cuijpers et al. [32] utilized data from the NEMESIS to investigate the relationship between incident ADs 
(expressed as incident rate ratios) and past smoking status. Smoking at 1-year follow-up was associated with increased incidence of grouped ADs (IRR 1.77 (1.10 to 2.86)) and GAD (IRR 3.80 (1.09 to 13.21)) after controlling for demographics and other risk factors. No other relationships were found (see Additional file 2, Table S2).

\section{Quasiprospective studies}

(A summary of this section is as follows: a small number of studies have utilized a single time point analysis and retrospective self-report patient data to draw quasiprospective associations between smoking, ND and ADs. These studies generally indicate increased smoking behaviors or nicotine dependence in individuals with pre-existing anxiety disorders, and vice versa, although studies are limited by the retrospective nature of data.)

\section{Baseline anxiety disorders and risk of smoking or nicotine dependence}

See also Additional file 3, Table S3. Breslau et al. [35] examined the 4,411 participants who completed the tobacco supplement of the NCS to assess the interaction between smoking, ND and DSM-III-R ADs, utilizing discrete time survival models with ADs as time dependent variables and controlling for race, gender, education and age. The onset of daily smoking was the age at which respondents first smoked daily for $\geq 1$ month. Increased odds for daily smoking were found in patients with preexisting (OR 1.9 (1.05 to 3.7)) or currently active GAD (OR 2.1 (1.1 to 3.9)), pre-existing (OR 1.6 (1.3 to 1.8$)$ ) or currently active specific phobia (OR 1.5 (1.3 to 1.8$)$ ), preexisting (OR 1.5 (1.2 to 1.7)) or currently active SP (OR 1.3 (1.1 to 1.6$)$ ), and pre-existing (OR 2.1 (1.6 to 2.9)) or currently active PTSD (OR 2.0 (1.4 to 2.9)).

Breslau et al. [35] further investigated the odds of smoking persistence and the transitioning from daily smoking to ND based upon pre-existing and currently active ADs. Increased odds of transitioning from daily smoking to ND were found in individuals with pre-existing and currently active agoraphobia, specific phobia, SP and PTSD. Interestingly, having pre-existing, but not currently active, PD was also strongly associated with increased odds of daily smoking to ND transition (OR 5.8 (3.0 to 11.6)). No associations existed between odds of smoking persistence and any pre-existing $\mathrm{AD}$.

Koenen et al. [36] utilized data from the national Vietnam Era Twin (VET) registry and retrospective self-report of age of onset to test prospective onset associations between DSM-III-R ADs and ND. Associations were controlled for various demographic and other risk factors and time-dependent covariates (conduct disorder, MDD, alcohol and drug abuse or dependence) were entered into models. The results demonstrated that pre-existing PTSD was associated with increased odds of subsequent ND (OR 1.73 (1.38 to 2.17)). Utilizing the Greater Smoky Mountain Study (GSMS), a longitudinal representative study of 4,500 children (aged 9 years, 11 years and 13 years) from western North Carolina, USA, Costello et al. [37] found children, both boys and girls, with any AD were more likely to commence smoking than those without an AD (see Additional file 3, Table S3).

\section{Smoking and nicotine dependence and risk of incident anxiety disorders}

See also Additional file 4, Table S4. Breslau et al. [38] utilized the NCS, including respondent recall about their age of smoking and ND onset, to assess the effect of these parameters on developing ADs. Adjusting for demographic characteristics, pre-existing daily smoking (defined as onset > 1 year prior to disorder onset) was associated with increased odds of PD (OR 2.6 (1.2 to $5.4)$ ) and agoraphobia (OR 4.4 (2.3 to 8.2)). The role of ND was assessed across all ADs. In this analysis, ND smokers and non-ND smokers maintained increased odds of PD and agoraphobia, but no other ADs. The only other associations were found in relation to past smokers (without ND) who exhibited decreased odds of PTSD (OR 0.2 (0.1 to 0.5)) when controlled for demographics and other pre-existing psychiatric disorders. Breslau et al. [38] extended their study by comparing the age of smoking onset (early vs not early; see Additional file 4, Table S4 for definitions), standardized pack years of smoking and time since quitting against odds of AD diagnosis. No association was found between early onset smoking and ADs, but increased years since quitting was associated with decreased odds of subsequent PD (OR 0.5 (0.4 to $0.7)$ ), agoraphobia (OR 0.5 (0.5 to 0.8$)$ ) and SP (OR 0.6 $(0.4$ to 0.8$))$. The associations between standardized pack years of smoking were not significant in all ADs except PD, where increased pack years of smoking appeared protective in current smokers but a risk factor in past smokers, and GAD where increased pack years was associated with increased odds of GAD in both current and past smokers.

In a separate analysis utilizing a subsample of NCS data, Breslau et al. [19] investigated the interaction between smoking characteristics and subsequent onset of PD. Significant relationships were discovered between prior daily smoking (HR 2.93 (1.84 to 4.66)) and smoking persistence in prior daily smokers (HR 3.18 (1.99 to 5.10)) and subsequent onset of PD. In addition, pre-existing ND was associated with increased odds of subsequent PTSD onset (OR 2.24 (1.78 to 2.83$)$ ) in the aforementioned study drawn from the VET registry [36].

\section{Cross-sectional studies}

(A summary of this section is as follows (see also Additional file 5, Table S5): a large number of studies have 
reported cross-sectional relationships between cigarette smoking, nicotine dependence and anxiety disorders. Many demonstrate higher rates of smoking and nicotine dependence in those with anxiety disorders, and vice versa. However, their utility is limited due to their inherent inability to provide insight into direction of causality.)

Almost all studies included in this review reported cross-sectional associations between smoking and/or ND and ADs. Studies providing cross-sectional information $[6,7,17,19,23,27,28,32,39-67]$ are listed in Additional file 5, Table S5. Descriptions of some selected larger studies utilizing population-based data are detailed below.

\section{Smoking or nicotine dependence by anxiety disorder status}

Lasser et al. [59] utilized the NCS to demonstrate increased rates of current and lifetime smoking in respondents with current and lifetime SP $(39.5 \%$ and $54 \%$ ), agoraphobia (38.4\% and 58.9\%), PD (35.9\% and $61.3 \%)$, specific phobia (40.3\% and $57.8 \%)$, PTSD $(45.3 \%$ and $63.3 \%$ ) and GAD (46\% and 68.4\%) when controlling for gender, age and geographical region. Utilizing the NCS-R data, Cougle et al. [44] explored the role of comorbidity in the association between ADs and smoking behavior. After controlling for demographics, depression and drug abuse/dependence, associations between increased odds of lifetime and 12-month daily smoking were observed with PTSD (Lifetime: OR 1.58 (1.21 to 2.06); 12-month: OR 1.46 (1.08 to 1.97)), 12-month daily smoking with PD (OR 1.42 (1.04 to 1.94)), and Lifetime daily smoking with GAD (OR 1.23 (1.05 to 1.61)).

In a nationally representative sample of the New Zealand population, individuals with ADs (grouped) had a smoking prevalence of $30.4 \%$ (27.7 to 33.0), and consumed approximately $16 \%$ of all cigarettes in New Zealand [7]. In Australia, data from the nationally representative National Survey of Mental Health and Wellbeing 2007 reported rates of current and daily smoking in those with individual ADs (range for current smoker: $33 \%$ to $45 \%$; range for daily smoker: $27 \%$ to $42 \%)$ well above the rates in respondents not reporting a mental disorder (current: 13.6\%; daily: 10.8\%) [6].

Cougle et al. [44] demonstrated increased odds of ND in patients with SP (OR 1.31 (1.01 to 1.71)), GAD (OR 1.59 (1.21 to 1.98$)$ ) and PTSD (OR 1.47 (1.01 to 2.16)) when adjusting for demographics, depression and drug abuse/dependence. Utilizing the NESARC for adults aged 18 to 25 years [46], the odds of 12 -month ND were significantly increased for respondents with lifetime specific phobia (OR 1.8 (1.16 to 2.88)) after controlling for other psychiatric disorders, smoking and demographic characteristics.

\section{Anxiety disorder by smoking or nicotine dependence status}

Utilizing the German Transitions in Alcohol Consumption and Smoking study, Schumann et al. [64] calculated unadjusted odds ratios for individual ADs based on smoking and ND status. When comparing ND ever smokers (respondents with ND who had smoked at least one cigarette daily for $\geq 4$ weeks at some point in their life) to nonND ever smokers, increased odds were found for PD (OR 2.92 (1.78 to 2.73$)$ ), SP (OR 3.07 (1.70 to 5.57$)$ ), specific phobia (OR 2.09 (1.63 to 2.68)), GAD (OR 4.26 (1.85 to 9.84)) and PTSD (OR 2.08 (1.13 to 3.83)). Grant et al. [52] used data from NESARC to compare the odds of ADs on ND status. Unadjusted odds ratios with individual ADs as dependent variables were greater across all assessed ADs. Point estimates for odds ratios ranged from 2.6 for SP to 4.6 for PD with agoraphobia for respondents with versus without ND. Data from the UK National Households Survey [49] demonstrated increased rates of GAD (4.1\% vs $2.4 \%)$, specific phobia (1.5\% vs $0.8 \%)$ and PD (1.5\% vs $0.5 \%)$ in respondents with ND versus those without ND. Degenhardt et al. [45] utilized the 1997 National Survey of Mental Health and Wellbeing in Australia to demonstrate increased odds of ADs (grouped) in current smokers versus never smokers (OR 1.50 (1.21 to 1.87)) when adjusted for demographic status, other drug use and neuroticism.

\section{Discussion}

Our systematic review of the literature revealed a total of 17 studies that provided prospective or quasiprospective information regarding the relationship between ADs, ND and cigarette smoking. Of these studies 14 provided prospective or quasiprospective information on the role of ADs on the risk of smoking or ND, and 7 studies provided the role of daily smoking or ND on onset of ADs. A further 31 studies reported only cross-sectional relationships between smoking, ND and ADs. The association between smoking, ND and ADs could be explained by three non-mutually exclusive relationships; smoking and ND leads to increased ADs, the reverse association, or a shared vulnerability model where a factor or group of factors increase smoking, ND and AD expression.

\section{Are anxiety disorders a risk factor for smoking onset?}

A relatively common hypothesis links increased anxiety with smoking onset and increased smoking behaviors. Multiple studies conducted in both general and clinical populations have demonstrated increased rates of smoking amongst individuals with all AD subtypes [68-70], although exceptions exist [71]. The results of these prospective, quasiprospective and cross-sectional studies are inconsistent, and importantly, many of these studies have failed to take into account the important role of 
comorbid substance use disorders. In a study of patients with ADs where those with alcohol and substance use disorders were excluded, rates of smoking were lower than the control population [72], indicating comorbid illness is important in understanding this association.

This review found a limited and heterogeneous literature evaluating the prospective effect of ADs on smoking onset in random population samples. The available data varied significantly on population characteristics (whole population vs specific age range), length of prospective follow-up (1 year to 10 years), diagnostic tool utilized and smoking definition. On balance, the 10-year followup of the National Comorbidity Survey [34] represents the likely best evidence available to date. From this analysis, baseline PD, SP, GAD and specific phobia, but not PTSD or agoraphobia, were associated with increased odds of commencing daily smoking. There are however important considerations in interpreting this study. First, the diagnostic classification system utilized in the first NCS (DSM-III-R) was updated in the NCS2 (DSM-IV). Although there is a large concordance between these diagnostic classifications across most ADs, some significant differences exist, particularly in the criteria of PTSD, which may have altered relative prevalence rates [73]. Second, and most importantly, the presented analyses did not control for the presence of other psychiatric disorders or for alcohol or drug abuse/dependence. As noted by Cougle et al. [44], ADs are often comorbid disorders, particularly with MDD and substance abuse disorders. In other presented prospective studies, univariate relationships between PD and smoking onset failed to sustain significance when comorbid MDD was included in the analytic models [19], and the presence of a comorbid substance use disorder reversed the direction of effect of baseline AD from protective to causative in the NESARC cohort [33]. The NCS follow-up revealed larger increased odds of daily smoking onset in respondents with alcohol or other substance abuse/dependence (OR point estimates between 2.6 and 4.2). As such, it is probable that some of the association between ADs and smoking is due to effects of comorbid substance abuse disorders.

Considering that most of the other available prospective studies failed to find significant associations between ADs and subsequent onset of smoking, it is currently difficult to draw definitive conclusions regarding $\mathrm{AD}$ as a risk factor for smoking. One potential factor that should be taken into account is the populations considered in the available literature. Population-based studies suggest that the average age individuals first use cigarettes has been declining, and now ranges between 12 years and 16 years [74]. In addition, smokers who commence earlier are more likely to persist than those that commence at a later age [75]. Given that most of the included studies assessed adult populations, it is possible many participants with ADs were already smoking and hence new onset smoking was relatively rare in assessed populations. In contrast to cross-sectional analyses, very limited prospective information is available on varying characteristics of smoking behavior (for example, persistence) and ADs in the available prospective studies.

Some studies have used a quasiprospective design, pairing cross-sectional data with retrospective self-report data, to assess the impact of ADs on smoking status. Such strategies are limited by recall bias, and any conclusions drawn must consider this. Analysis of the NCS by Breslau et al. [35] found increased odds of daily smoking in those with a variety of pre-existing or current ADs. However, these analyses did not control for the effects of comorbid substance use disorders or other psychiatric disorders and, additionally, were limited to onset of daily smoking as opposed to smoking more generally. When considering the effects of pre-existing ADs on smoking persistence, no significant associations were found.

A number of theories are proposed to explain why ADs may lead to increased rates of smoking. Theoretical explanations are informed by both psychological (for example, conditioning theory, cognitive theory, anxiety sensitivity [76]) and biological (for example, nicotine effects and withdrawal) factors. One explanatory model relates to the use of cigarettes as an anxiolytic agent (that is, self-treatment). Evidence supports that nicotine exposure does produce a subjective calming effect, although this is coupled with an increase in objective measures of physiological arousal [77]. Tachyphylaxis and homeostatic adjustment however implies that acute and chronic effects of cigarettes, as with any agents that induce tolerance and dependence, may differ substantially. Treatment of this extensive literature is beyond this review, although we refer readers to a number of excellent comprehensive reviews on this subject [78-81], which include identification of the methodological challenges in exploring the effect of anxiety traits and states on smoking behaviors.

\section{Are anxiety disorders a risk factor for later nicotine dependence?}

Inconsistent although clearer associations between baseline ADs and subsequent ND were found in the literature. In the 10-year follow-up of the NCS [34], having baseline PTSD, agoraphobia and specific phobia were associated with increased odds of onset of ND amongst already smokers, and PTSD, SP and specific phobia were associated with increased odds for ND amongst the whole population. The clear association between baseline PTSD and ND was replicated by Breslau et al. [21] in the Detroit Epidemiologic Study, while Isensee et al. [27] found baseline PD to be associated with increased risk for subsequent ND. Given anxiety disorders are associated with negative affect [82], and negative affect is linked to increased 
smoking motivation [83], it is possible that negative affect may underpin an increased likelihood to developing ND in those with ADs. Further evidence demonstrating an effect of duration of anxiety disorder diagnosis and later ND severity would be useful.

\section{Is smoking or nicotine dependence a risk factor for onset of anxiety disorders?}

A limited number of prospective studies are available assessing smoking or ND as risk factors for incident ADs in population-based epidemiological studies. The available literature supports ND as increasing the odds of later PTSD, and smoking as increasing the odds for some (PD and GAD), but not all, ADs and interpretation of some analyses are complicated by non-inclusion of potential confounders. For example, the prospective odds of PD onset were increased in the Oregon Adolescent Depression Project, the New York Adolescent Cohort and the EDSP in individuals with a history of prior smoking versus non-smokers. This result remained significant when controlling for other ADs in the Oregon Adolescent Depression Study, but failed to maintain significance when comorbid psychiatric disorders were controlled for in the EDSP study. However, smoking as a risk factor for PD is supported by the Detroit Epidemiologic Study, which found increased risk of PD onset in prior daily smokers, with a stronger association in prior daily smokers who continued to smoke. These analyses controlled for MDD, but not other psychiatric or substance use disorders. The use of quasiprospective methodology on data from the NCS allowed further exploration of this relationship. These data suggested that daily smoking was a risk factor for increased odds of PD and agoraphobia, with analyses controlling for other psychiatric disorders including substance use disorders [38]. A demonstrated dose-dependent relationship would support a causal association between cigarette smoking and subsequent onset of ADs. Breslau demonstrated that increased standardized pack years of smoking were associated with increased odds of GAD, but decreased odds of PD [38] in dose dependent fashion.

The interaction between panic, PD and cigarette smoking has received more investigation in epidemiological and laboratory-based studies than other ADs, and the evidence was recently reviewed [84]. The literature has demonstrated that earlier age of smoking initiation is associated with increased risk of PD [85], and smokers demonstrated earlier PD onset in comparison to nonsmokers [86]. Given the somatic nature of panic attack symptomatology, it is possible that smoking may be more likely to produce panic-type AD symptoms secondary to the physical effects of smoking (for example, respiratory and autonomic disturbance). Panic is influenced by respiratory sensitivity, and nicotine alters the sensitivity set point of cholinergic respiratory neurons [87]. From their review of the literature, Cosci et al. [84] nominated three potential hypotheses underpinning the association between cigarette smoking and panic. The first is a 'moderational model', whereby neuroticism moderates the panic/smoking association. The second is a 'pathoplastic model', where smoking influences PD expression by 'exacerbating affective disturbances and negative health process' [88], including a combination of direct negative health effects (for example, induction of chronic obstructive pulmonary disease (COPD)), acute physiological effects (for example, increased noradrenaline and autonomic sensations) and negative self-perception of health status [89]. The third is the 'false suffocation alarm' theory, proposed by Klein [90], who suggested that smoking may induce hypersensitivity to suffocation signals and increase the risk of experiencing panic. The suffocation false alarm may potentially be mediated by episodic dysfunction in regulation of endogenous opioid function [91].

A number of studies have assessed the interaction of smoking and PTSD. A population-based follow-up investigation of individuals affected by a fireworks factory explosion in The Netherlands found that those who were smokers at time of traumatic incident had an increased chance of experiencing severe anxiety symptoms (OR 2.32 (1.19 to 4.53)) and disaster related PTSD (OR 2.64 (1.05 to 6.62)) at 4-year follow-up, controlling for baseline symptoms, demographic characteristics and life events [92]. This supports the results of Koenen et al. [36], who demonstrated that the presence of ND increased the risk of developing PTSD in trauma exposed men (HR 1.98 (1.61 to 2.42)) and that shared genetic effects explained $63 \%$ of the ND-PTSD association. A number of neurobiological (for example, alterations to the hypothalamic-pituitary-adrenal axis, sympathetic nervous system hyperactivity alteration to neurotransmitter system functioning) and psychological factors may contribute to a PTSD, ND and cigarette smoking association (see Fu et al. [93] for review). ND may increase ADs by exacerbating anxiety responses [94], or by facilitating other psychiatric states (for example, depression) that may predispose individuals to develop anxiety. In addition, the number of ND symptoms and nicotine withdrawal symptoms has been robustly correlated in a dose-dependent fashion with increasing anxiety disorder diagnosis [56]. In reference to other ADs, supportive evidence was found for smoking increasing the odds of developing GAD, while no evidence was found to support smoking as a risk factor for onset of $\mathrm{SP}$, although some evidence supports the reverse association in female ND smokers [28].

In assessing smoking or ND as a risk factor for ADs, one factor that must be considered is that ADs have been estimated to exhibit a median age of onset of only 
6 years [95]. Given the early age of AD development, from early childhood through adolescence, exposure to environmental cigarette smoke may influence AD development. Exposure to second-hand smoke has been demonstrated to be positively associated with symptoms of GAD, but not PD in children and adolescents age 8 years to 15 years [96]. Additionally, early onset smoking (10 to 15 years) has been associated with an earlier onset of ADs when compared to late onset smoking (> 15 years) [97]. It is possible that exposure to cigarette smoke in critical periods of brain development may be one factor that predisposes individuals to subsequent develop ADs. A number of biological pathways may underpin this effect, including alteration in neurotransmitter function, and induction of oxidative and nitrosative stress that may overwhelm intrinsic defenses inhibiting normal neuronal cell functions, including neuroplasticity and neurogenesis.

\section{Shared vulnerability between smoking, ND and ADs}

A number of shared vulnerability factors contribute to cigarette smoking behavior and ADs, including early childhood experiences and environment $[83,98,99]$, problems with impulsivity [100] and distress tolerance and neurotic personality traits $[101,102]$. Indeed, it is possible that these shared vulnerability factors are further underpinned by other factors (for example, genetics, fetal insults), and ADs and cigarette smoke could alter their expression (for example, cigarette smoking may reinforce poor distress tolerance). For a more detailed discussion of the role and interaction of shared vulnerability factors see the review by Morissette et al. [79].

\section{Limitations and directions for future research}

It is important to consider the limitations of this study design in interpreting the results. This review was designed deliberately to only consider random, large general population samples where validated structured diagnostic tools were utilized to make diagnoses. Effort was made to identify all studies meeting inclusion criteria, although it is possible some important sources of information were not identified, particularly from non-English language journals. The inclusion criteria were designed to allow for selection of only the best quality evidence not restricted by certain biases (for example, selection bias, recall bias) although as consequence of this a number of identified studies involving samples drawn from clinic populations [72,103-105] and studies which utilized anxiety symptom scores [8] or non-validated diagnostic tools (for example, self-report) as end points were excluded from final analysis. The heterogeneous nature of included studies, particularly in regards to population demographics; smoking definition; diagnostic criteria utilized; diagnostic group included; and comorbid factors included in analysis also complicates interpretation. A number of future studies would assist in clarifying issues from this review. First, the continuation of current prospective cohorts over longer periods and the recruitment of new cohorts, incorporating and measuring changes to smoking behavior over time, would assist in clarifying the relationship between smoking and ADs. Similarly, given the early onset nature of many ADs, maternal and birth cohorts assessing these issues would assist greatly in deepening our understanding. Exploration of the effects of quitting on anxiety and depression would also clarify associations. In addition, further exploration of potential biological mechanisms underpinning associated effects of smoking and ADs would help to elucidate shared vulnerabilities underpinning both ADs and smoking.

\section{Conclusions}

A large literature has demonstrated that individuals with ADs have higher rates of smoking and ND than those without ADs, although most extant information is drawn from cross-sectional studies. In terms of prospective studies, there exists a limited heterogeneous literature examining the relationship between cigarette smoking, ND and ADs in population-based epidemiological studies utilizing structured diagnostic tools linked to ICD or DSM criteria. The available prospective evidence provides support for smoking and ND as being risk factors for the onset of PD and GAD, although varied results are found across studies. In addition, some ADs have been associated with subsequent onset of smoking and ND, although many studies failed to include comorbid substance use disorders and other psychiatric disorders in analyses.

\section{Additional material}

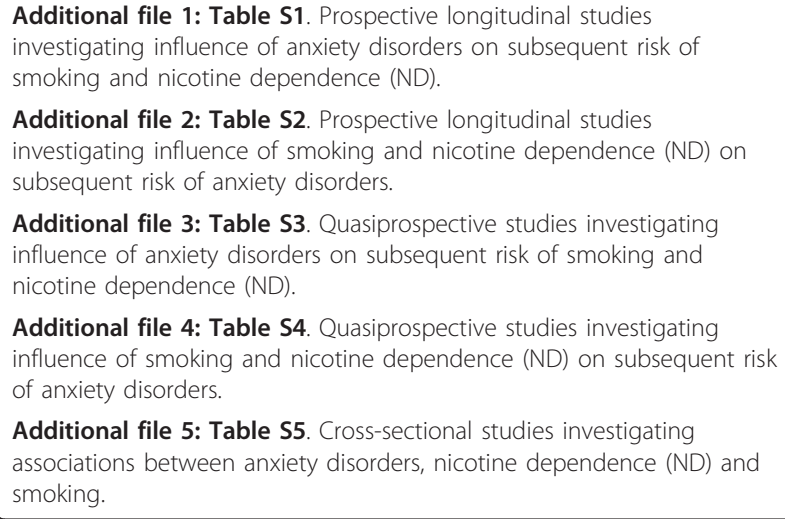

Additional file 3: Table S3. Quasiprospective studies investigating influence of anxiety disorders on subsequent risk of smoking and nicotine dependence (ND)

Additional file 4: Table S4. Quasiprospective studies investigating influence of smoking and nicotine dependence (ND) on subsequent risk of anxiety disorders.

Additional file 5: Table S5. Cross-sectional studies investigating associations between anxiety disorders, nicotine dependence (ND) and smoking.

\section{Acknowledgements}

The authors wish to thank Professor Dan Stein and Professor Simon $\varnothing v e r l a n d$ for their insightful comments and suggestions, which greatly improved this manuscript. 


\section{Author details}

'Deakin University School of Medicine, Barwon Health, Geelong, Victoria, Australia. ${ }^{2}$ Department of Psychiatry, Melbourne University, Parkville, Victoria, Australia. ${ }^{3}$ NorthWest Academic Centre, Department of Medicine, The University of Melbourne, St Albans, Victoria, Australia. ${ }^{4}$ Orygen Youth Health Research Centre, Centre for Youth Mental Health, Parkville, Victoria, Australia. ${ }^{5}$ The Florey Institute for Neuroscience and Mental Health, Parkville, Victoria, Australia.

\section{Authors' contributions}

SM conceived of the study, developed the methods, conducted the literature search and led the development of the final manuscript. FNJ, JAP and $\mathrm{MB}$ contributed to the study design and data analysis, and provided intellectual content to the final manuscript. All authors read and approved the final manuscript.

\section{Competing interests}

SM declares no conflicts of interest in relation to this article. FNJ has received grant/research support from the Brain and Behaviour Research Institute, the National Health and Medical Research Council, Australian Rotary Health, the Geelong Medical Research Foundation, the lan Potter Foundation, Eli Lilly and The University of Melbourne and has been a paid speaker for Sanofi-Synthelabo, Janssen Cilag and Eli Lilly. She is supported by an NHMRC Training Fellowship (\#628912). JAP has received speaker fees from Amgen, Eli Lilly and Sanofi-Aventis and funding from the Geelong Region Medical Research Foundation, Barwon Health, Perpetual Trustees, the Dairy Research and Development Corporation, The University of Melbourne, the Ronald Geoffrey Arnott Foundation, ANZ Charitable Trust, the American Society for Bone and Mineral Research, Amgen (Europe) GmBH and the NHMRC. MB has received Grant/Research Support from the NIH, Cooperative Research Centre, Simons Autism Foundation, Cancer Council of Victoria, Stanley Medical Research Foundation, MBF, NHMRC, Beyond Blue, Geelong Medical Research Foundation, Bristol Myers Squibb, Eli Lilly, Glaxo SmithKline, Organon, Novartis, Mayne Pharma and Servier, has been a speaker for Astra Zeneca, Bristol Myers Squibb, Eli Lilly, Glaxo SmithKline, Janssen Cilag, Lundbeck, Merck, Pfizer, Sanofi Synthelabo, Servier, Solvayand Wyeth, and served as a consultant to Astra Zeneca, Bristol Myers Squibb, Eli Lilly, Glaxo SmithKline, Janssen Cilag, Lundbeck and Servier.

Received: 30 April 2012 Accepted: 19 October 2012

Published: 19 October 2012

\section{References}

1. McEvoy PM, Grove R, Slade T: Epidemiology of anxiety disorders in the Australian general population: findings of the 2007 Australian National Survey of Mental Health and Wellbeing. Aust NZ J Psychiatry 2011, 45:957-967.

2. Kessler RC, Chiu WT, Demler O, Merikangas KR, Walters EE: Prevalence, severity, and comorbidity of 12-month DSM-IV disorders in the National Comorbidity Survey Replication. Arch Gen Psychiatry 2005, 62:617-627.

3. Mendlowicz MV, Stein MB: Quality of life in individuals with anxiety disorders. Am J Psychiatry 2000, 157:669-682.

4. Stein MB, Roy-Byrne PP, Craske MG, Bystritsky A, Sullivan G, Pyne JM, Katon W, Sherbourne CD: Functional impact and health utility of anxiety disorders in primary care outpatients. Med Care 2005, 43:1164-1170.

5. Lawrence D, Mitrou F, Zubrick SR: Smoking and mental illness: results from population surveys in Australia and the United States. BMC Public Health 2009, 9:285.

6. Lawrence D, Considine J, Mitrou F, Zubrick SR: Anxiety disorders and cigarette smoking: Results from the Australian Survey of Mental Health and Wellbeing. Aust NZ J Psychiatry 2010, 44:520-527.

7. Tobias $M$, Templeton $R$, Collings $S$ : How much do mental disorders contribute to New Zealand's tobacco epidemic? Tob Control 2008, 17:347-350.

8. Mykletun A, Overland S, Aaro LE, Liabo HM, Stewart R: Smoking in relation to anxiety and depression: evidence from a large population survey: the HUNT study. Eur Psychiatry 2008, 23:77-84.

9. Iniguez SD, Warren BL, Parise EM, Alcantara LF, Schuh B, Maffeo ML, Manojlovic Z, Bolanos-Guzman CA: Nicotine exposure during adolescence induces a depression-like state in adulthood. Neuropsychopharmacology 2009, 34:1609-1624.
10. Zvolensky MJ, Schmidt NB, McCreary BT: The impact of smoking on panic disorder: an initial investigation of a pathoplastic relationship. J Anxiety Disord 2003, 17:447-460.

11. Patton GC, Carlin JB, Coffey C, Wolfe R, Hibbert M, Bowes G: Depression, anxiety, and smoking initiation: a prospective study over 3 years. Am J Public Health 1998, 88:1518-1522.

12. Tjora T, Hetland J, Aaro LE, Overland S: Distal and proximal family predictors of adolescents' smoking initiation and development: a longitudinal latent curve model analysis. BMC Public Health 2011, 11:911.

13. Schaap MM, Kunst AE: Monitoring of socio-economic inequalities in smoking: learning from the experiences of recent scientific studies. Public Health 2009, 123:103-109.

14. American Psychiatric Association: Diagnostic and Statistical Manual of Mental Disorders. 4 edition. Washington, DC: American Psychiatric Association; 2000, text revision.

15. World health Organization: International Classifications of Diseases (ICD). [http://www.who.int/classifications/icd/en/].

16. Orvaschel H, Puig-Antich J, Chambers W, Tabrizi MA, Johnson R Retrospective assessment of prepubertal major depression with the Kiddie-SADS-e. J Am Acad Child Psychiatry 1982, 21:392-397.

17. Brown RA, Lewinsohn PM, Seeley JR, Wagner EF: Cigarette smoking, major depression, and other psychiatric disorders among adolescents. J Am Acad Child Adolesc Psychiatry 1996, 35:1602-1610.

18. Goodwin RD, Lewinsohn PM, Seeley JR: Cigarette smoking and panic attacks among young adults in the community: the role of parental smoking and anxiety disorders. Biol Psychiatry 2005, 58:686-693.

19. Breslau N, Klein DF: Smoking and panic attacks: an epidemiologic investigation. Arch Gen Psychiatry 1999, 56:1141-1147.

20. Robins L, Helzer J, Cotler L, Golding E: NIMH Diagnostic Interview Schedule Version III Revised Rockville, MD: National Institute of Mental Health; 1989

21. Breslau N, Davis GC, Schultz LR: Posttraumatic stress disorder and the incidence of nicotine, alcohol, and other drug disorders in persons who have experienced trauma. Arch Gen Psychiatry 2003, 60:289-294.

22. Johnson JG, Cohen P, Pine DS, Klein DF, Kasen S, Brook JS: Association between cigarette smoking and anxiety disorders during adolescence and early adulthood. JAMA 2000, 284:2348-2351.

23. Costello E, Edelbrock C, Duncan M, Kalas R: Testing of the NIMH Diagnostic Interview Schedule for Children (DISC) in a Clinical Population: Final Report to the Center for Epidemiological Studies, NIMH Pittsburgh, PA: University of Pittsburgh; 1984

24. Max Planck Institute of Psychiatry: Early Developmental Stages of Psychopathology (EDSP) study. [http://www.mpipsykl.mpg.de/en/research/ themes/depression/ising_01/index.html].

25. In DA-X-Interviews: Manual fur Screening-Verfahren und Interview; Interviewheft La ngsschnittuntersuchung (DIA-X- Lifetime); Erganzungsheft (DIAX-Lifetime); Intenviewheft Querschnittuntersuchung (DIA-X-12 Monate); Erganzungsheft (DIA-X-12Monate); PC-Programm zur Durchfuhrung des Interviews (Langsund Querschnittuntersuchung); Auswertungsprogramm. Edited by: Wittchen H-U, Pfister H. Frankfurt, Germany: Swets 1997.

26. World Health Organization: Composite International Diagnostic Interview (CIDI) Geneva, Switzerland: WHO; 1990.

27. Isensee B, Wittchen HU, Stein MB, Hofler M, Lieb R: Smoking increases the risk of panic: findings from a prospective community study. Arch Gen Psychiatry 2003, 60:692-700.

28. Sonntag H, Wittchen HU, Hofler M, Kessler RC, Stein MB: Are social fears and DSM-IV social anxiety disorder associated with smoking and nicotine dependence in adolescents and young adults? Eur Psychiatry 2000, 15:67-74.

29. Woodward L, Fergusson DM: Life course outcomes of young people with anxiety disorders in adolescence. J Am Acad Child Adolesc Psychiatry 2001, 40:1086-1093.

30. Chou KL, Mackenzie CS, Liang K, Sareen J: Three-year incidence and predictors of first-onset of DSM-IV mood, anxiety, and substance use disorders in older adults: results from Wave 2 of the National Epidemiologic Survey on Alcohol and Related Conditions. J Clin Psychiatry 2011, 72:144-155.

31. Grant B, Dawson D, Hasin D: The Wave 2 National Epidemiologic Survey on Alcohol and Related Conditions Alcohol Use Disorder and Associated Disabilities Interview Schedule DSM-IV Version Bethesda, MD: National Institutes of Health; 2004. 
32. Cuijpers P, Smit F, ten Have M, de Graaf R: Smoking is associated with first-ever incidence of mental disorders: a prospective population-based study. Addiction 2007, 102:1303-1309.

33. Johnson EO, Novak SP: Onset and persistence of daily smoking: the interplay of socioeconomic status, gender, and psychiatric disorders. Drug Alcohol Depend 2009, 104(Suppl 1):S50-57.

34. Swendsen J, Conway KP, Degenhardt L, Glantz M, Jin R, Merikangas KR, Sampson N, Kessler RC: Mental disorders as risk factors for substance use, abuse and dependence: results from the 10-year follow-up of the National Comorbidity Survey. Addiction 2010, 105:1117-1128.

35. Breslau N, Novak SP, Kessler RC: Psychiatric disorders and stages of smoking. Biol Psychiatry 2004, 55:69-76.

36. Koenen KC, Hitsman B, Lyons MJ, Niaura R, McCaffery J, Goldberg J, Eisen SA, True W, Tsuang M: A twin registry study of the relationship between posttraumatic stress disorder and nicotine dependence in men. Arch Gen Psychiatry 2005, 62:1258-1265.

37. Costello EJ, Erkanli A, Federman E, Angold A: Development of psychiatric comorbidity with substance abuse in adolescents: effects of timing and sex. J Clin Child Psychol 1999, 28:298-311.

38. Breslau N, Novak SP, Kessler RC: Daily smoking and the subsequent onset of psychiatric disorders. Psychol Med 2004, 34:323-333.

39. Acierno R, Kilpatrick DG, Resnick H, Saunders B, De Arellano M, Best C: Assault, PTSD, family substance use, and depression as risk factors for cigarette use in youth: findings from the National Survey of Adolescents. J Trauma Stress 2000, 13:381-396.

40. Acierno RA, Kilpatrick DG, Resnick HS, Saunders BE, Best CL: Violent assault, posttraumatic stress disorder, and depression. Risk factors for cigarette use among adult women. Behav Modif 1996, 20:363-384.

41. Breslau N: Psychiatric comorbidity of smoking and nicotine dependence. Behav Genet 1995, 25:95-101.

42. Breslau N, Kilbey M, Andreski P: Nicotine dependence, major depression, and anxiety in young adults. Arch Gen Psychiatry 1991, 48:1069-1074.

43. Carroll D, Phillips AC, Thomas GN, Gale CR, Deary I, Batty GD: Generalized anxiety disorder is associated with metabolic syndrome in the Vietnam experience study. Biol Psychiatry 2009, 66:91-93.

44. Cougle JR, Zvolensky MJ, Fitch KE, Sachs-Ericsson N: The role of comorbidity in explaining the associations between anxiety disorders and smoking. Nicotine Tob Res 2010, 12:355-364.

45. Degenhardt $L$, Hall W: The relationship between tobacco use, substanceuse disorders and mental health: Results from the National Survey of Mental Health and Well-being. Nicotine Tob Res 2001, 3:225-234.

46. Dierker L, Donny E: The role of psychiatric disorders in the relationship between cigarette smoking and DSM-IV nicotine dependence among young adults. Nicotine Tob Res 2008, 10:439-446.

47. Dierker LC, Sledjeski EM, Botello-Harbaum M, Ramirez RR, Chavez LM, Canino G: Association between psychiatric disorders and smoking stages within a representative clinic sample of Puerto Rican adolescents. Compr Psychiatry 2007, 48:237-244.

48. Durai UN, Chopra MP, Coakley E, Llorente MD, Kirchner JE, Cook JM, Levkoff SE: Exposure to trauma and posttraumatic stress disorder symptoms in older veterans attending primary care: comorbid conditions and self-rated health status. J Am Geriatr Soc 2011, 59:1087-1092.

49. Farrell $M$, Howes $S$, Bebbington $P$, Brugha $T$, Jenkins $R$, Lewis $G$, Marsden J, Taylor C, Meltzer H: Nicotine, alcohol and drug dependence, and psychiatric comorbidity-results of a national household survey. Int Rev Psychiatry 2003, 15:50-56.

50. Glassman AH, Helzer JE, Covey LS, Cottler LB, Stetner F, Tipp JE, Johnson J: Smoking, smoking cessation, and major depression. JAMA 1990, 264:1546-1549.

51. Grabe HJ, Meyer C, Hapke U, Rumpf HJ, Freyberger HJ, Dilling H, John U: Lifetime-comorbidity of obsessive-compulsive disorder and subclinical obsessive-compulsive disorder in Northern Germany. Eur Arch Psychiatry Clin Neurosci 2001, 251:130-135.

52. Grant BF, Hasin DS, Chou SP, Stinson FS, Dawson DA: Nicotine dependence and psychiatric disorders in the United States: results from the national epidemiologic survey on alcohol and related conditions. Arch Gen Psychiatry 2004, 61:1107-1115.

53. Grant BF, Hasin DS, Stinson FS, Dawson DA, June Ruan W, Goldstein RB, Smith SM, Saha TD, Huang B: Prevalence, correlates, co-morbidity, and comparative disability of DSM-IV generalized anxiety disorder in the
USA: results from the National Epidemiologic Survey on Alcohol and Related Conditions. Psychol Med 2005, 35:1747-1759.

54. Gwynn RC, McQuistion HL, McVeigh KH, Garg RK, Frieden TR, Thorpe LE: Prevalence, diagnosis, and treatment of depression and generalized anxiety disorder in a diverse urban community. Psychiatr Serv 2008, 59:641-647.

55. Hapke U, Schumann A, Rumpf HJ, John U, Konerding U, Meyer C: Association of smoking and nicotine dependence with trauma and posttraumatic stress disorder in a general population sample. Journal Nerv Ment Dis 2005, 193:843-846.

56. John U, Meyer C, Hapke U, Rumpf HJ: Nicotine dependence and lifetime amount of smoking in a population sample. Eur J Public Health 2004, 14:182-185.

57. Kandel DB, Huang FY, Davies M: Comorbidity between patterns of substance use dependence and psychiatric syndromes. Drug Alcohol Depend 2001, 64:233-241.

58. Kandel DB, Johnson JG, Bird HR, Canino G, Goodman SH, Lahey BB, Regier DA, Schwab-Stone M: Psychiatric disorders associated with substance use among children and adolescents: findings from the Methods for the Epidemiology of Child and Adolescent Mental Disorders (MECA) Study. J Abnorm Child Psychol 1997, 25:121-132.

59. Lasser K, Boyd JW, Woolhandler S, Himmelstein DU, McCormick D, Bor DH: Smoking and mental illness: a population-based prevalence study. JAMA 2000, 284:2606-2610.

60. Morris CD, Giese AA, Turnbull JJ, Dickinson M, Johnson-Nagel N: Predictors of tobacco use among persons with mental illnesses in a statewide population. Psychiatr Serv 2006, 57:1035-1038.

61. Nelson $\mathrm{CB}$, Wittchen HU: Smoking and nicotine dependence. Results from a sample of 14- to 24-year-olds in Germany. Eur Addict Res 1998, 4:42-49.

62. Op Den Velde W, Aarts PG, Falger PR, Hovens JE, Van Duijn H, De Groen JH, Van Duijn MA: Alcohol use, cigarette consumption and chronic posttraumatic stress disorder. Alcohol Alcohol 2002, 37:355-361.

63. Schmitz N, Kruse J, Kugler J: Disabilities, quality of life, and mental disorders associated with smoking and nicotine dependence. Am J Psychiatry 2003, 160:1670-1676.

64. Schumann A, Hapke U, Meyer C, Rumpf HJ, John U: Prevalence, characteristics, associated mental disorders and predictors of DSM-IV nicotine dependence. Eur Addict Res 2004, 10:29-34.

65. Vesga-Lopez O, Schneier FR, Wang S, Heimberg RG, Liu SM, Hasin DS, Blanco C: Gender differences in generalized anxiety disorder: results from the National Epidemiologic Survey on Alcohol and Related Conditions (NESARC). J Clin Psychiatry 2008, 69:1606-1616.

66. Wittchen HU, Stein MB, Kessler RC: Social fears and social phobia in a community sample of adolescents and young adults: prevalence, risk factors and co-morbidity. Psychological Medicine 1999, 29:309-323.

67. Wu P, Goodwin RD, Fuller C, Liu X, Comer JS, Cohen P, Hoven CW: The relationship between anxiety disorders and substance use among adolescents in the community: specificity and gender differences. $J$ Youth Adolesc 2010, 39:177-188.

68. Hughes JR, Hatsukami DK, Mitchell JE, Dahlgren LA: Prevalence of smoking among psychiatric outpatients. Am J Psychiatry 1986, 143:993-997.

69. Pohl R, Yeragani VK, Balon R, Lycaki H, McBride R: Smoking in patients with panic disorder. Psychiatry Res 1992, 43:253-262.

70. Beckham JC, Roodman AA, Shipley RH, Hertzberg MA, Cunha GH, Kudler HS, Levin ED, Rose JE, Fairbank JA: Smoking in Vietnam combat veterans with post-traumatic stress disorder. J Trauma Stress 1995, 8:461-472

71. Bejerot S, Humble M: Low prevalence of smoking among patients with obsessive-compulsive disorder. Compr Psychiatry 1999, 40:268-272.

72. Baker-Morissette S, Gulliver S, Wiegel M, Barlow D: Prevalence of smoking in anxiety disorders uncomplicated by comorbid alcohol or substance abuse. J Psychopathol Behav Assess 2004, 26:107-112.

73. Van Ameringen M, Mancini C, Patterson B: The impact of changing diagnostic criteria in posttraumatic stress disorder in a Canadian epidemiologic sample. J Clin Psychiatry 2011, 72:1034-1041.

74. Schneider S, Mohnen SM, Pust S: The average age of smoking onset in Germany - trends and correlates. Int J Public Health 2008, 53:160-164.

75. Khuder SA, Dayal HH, Mutgi AB: Age at smoking onset and its effect on smoking cessation. Addict Behav 1999, 24:673-677.

76. Zvolensky MJ, Kotov R, Bonn-Miller MO, Schmidt NB, Antipova AV: Anxiety sensitivity as a moderator of association between smoking status and 
panic-related processes in a representative sample of adults. $J$ Psychiatr Res 2008, 42:69-77.

77. Perkins KA: Individual variability in responses to nicotine. Behav Genet 1995, 25:119-132.

78. Ameringer KJ, Leventhal AM: Applying the tripartite model of anxiety and depression to cigarette smoking: an integrative review. Nicotine Tob Res 2010, 12:1183-1194.

79. Morissette SB, Tull MT, Gulliver SB, Kamholz BW, Zimering RT: Anxiety, anxiety disorders, tobacco use, and nicotine: a critical review of interrelationships. Psychol Bull 2007, 133:245-272.

80. Zvolensky MJ, Bernstein A, Marshall EC, Feldner MT: Panic attacks, panic disorder, and agoraphobia: associations with substance use, abuse, and dependence. Curr Psychiatry Rep 2006, 8:279-285.

81. Zvolensky MJ, Feldner MT, Leen-Feldner EW, McLeish AC: Smoking and panic attacks, panic disorder, and agoraphobia: a review of the empirical literature. Clin Psychol Rev 2005, 25:761-789.

82. Watson D: Rethinking the mood and anxiety disorders: a quantitative hierarchical model for DSM-V. J Abnorm Psychol 2005, 114:522-536.

83. Baker TB, Brandon TH, Chassin L: Motivational influences on cigarette smoking. Annu Rev Psychol 2004, 55:463-491.

84. Cosci F, Knuts IJ, Abrams K, Griez EJ, Schruers KR: Cigarette smoking and panic: a critical review of the literature. J Clin Psychiatry 2010, 71:606-615.

85. Bernstein A, Zvolensky MJ, Schmidt NB, Sachs-Ericcson N: Developmental course(s) of lifetime cigarette use and panic attack comorbidity: an equifinal phenomenon? Behav Modif 2007, 31:117-135.

86. Amering M, Bankier B, Berger P, Griengl H, Windhaber J, Katschnig H: Panic disorder and cigarette smoking behavior. Compr Psychiatry 1999, 40:35-38

87. Coddou C, Bravo E, Eugenin J: Alterations in cholinergic sensitivity of respiratory neurons induced by pre-natal nicotine: a mechanism for respiratory dysfunction in neonatal mice. Philos Trans $R$ Soc Lond B Biol Sci 2009, 364:2527-2535.

88. Zvolensky MJ, Schmidt NB, McCreary BT: The impact of smoking on panic disorder: an initial investigation of a pathoplastic relationship. J Anxiety Disord 2003, 17:447-460.

89. Zvolensky M, Bernstein A: Cigarette smoking and panic psychopathology. Curr Direct Psychol Sci 2005, 14:301-305.

90. Klein DF: False suffocation alarms, spontaneous panics, and related conditions. An integrative hypothesis. Arch Gen Psychiatry 1993, 50:306-317.

91. Preter M, Klein DF: Panic, suffocation false alarms, separation anxiety and endogenous opioids. Prog Neuropsychopharmacol Biol Psychiatry 2008, 32:603-612.

92. Van der Velden PG, Grievink L, Olff M, Gersons BP, Kleber RJ: Smoking as a risk factor for mental health disturbances after a disaster: a prospective comparative study. J Clin Psychiatry 2007, 68:87-92.

93. Fu SS, McFall M, Saxon AJ, Beckham JC, Carmody TP, Baker DG, Joseph AM: Post-traumatic stress disorder and smoking: a systematic review. Nicotine Tob Res 2007, 9:1071-1084.

94. Jamal M, Willem Van der Does AJ, Cuijpers P, Penninx BW: Association of smoking and nicotine dependence with severity and course of symptoms in patients with depressive or anxiety disorder. Drug Alcohol Depend

95. Merikangas KR, He JP, Burstein M, Swanson SA, Avenevoli S, Cui L, Benjet C, Georgiades K, Swendsen J: Lifetime prevalence of mental disorders in U.S. adolescents: results from the National Comorbidity Survey ReplicationAdolescent Supplement (NCS-A). J Am Acad Child Adolesc Psychiatry 2010, 49:980-989.

96. Bandiera FC, Richardson AK, Lee DJ, He JP, Merikangas KR: Secondhand smoke exposure and mental health among children and adolescents. Arch Pediatr Adolesc Med 2011, 165:332-338.

97. Jamal M, Does AJ, Penninx BW, Cuijpers P: Age at smoking onset and the onset of depression and anxiety disorders. Nicotine Tob Res 2011, 13:809-819.

98. Goodwin RD, Fergusson DM, Horwood L: Association between anxiety disorders and substance use disorders among young persons: results of a 21-year longitudinal study. J Psychiatr Res 2004, 38:295-304.

99. Kassel JD, Stroud LR, Paronis CA: Smoking, stress, and negative affect: correlation, causation, and context across stages of smoking. Psychol Bull 2003, 129:270-304

100. Masse LC, Tremblay RE: Behavior of boys in kindergarten and the onset of substance use during adolescence. Arch Gen Psychiatry 1997, 54:62-68.
101. Byrne DG, Byrne AE, Reinhart Ml: Personality, stress and the decision to commence cigarette smoking in adolescence. J Psychosom Res 1995, 39:53-62.

102. Breslau N, Kilbey MM, Andreski P: Vulnerability to psychopathology in nicotine-dependent smokers: an epidemiologic study of young adults. Am J Psychiatry 1993, 150:941-946.

103. Bejerot S, von Knorring L, Ekselius L: Personality traits and smoking in patients with obsessive-compulsive disorder. Eur Psychiatry 2000, 15:395-401.

104. Lopes FL, Nascimento I, Zin WA, Valenca AM, Mezzasalma MA, Figueira I, Nardi AE: Smoking and psychiatric disorders: a comorbidity survey. Braz $J$ Med Biol Res 2002, 35:961-967.

105. Tilley S: Alcohol, other drugs and tobacco use and anxiolytic effectiveness. A comparison of anxious patients and psychiatric nurses. Br J Psychiatry 1987, 151:389-392.

\section{Pre-publication history}

The pre-publication history for this paper can be accessed here: http://www.biomedcentral.com/1741-7015/10/123/prepub

doi:10.1186/1741-7015-10-123

Cite this article as: Moylan et al: Cigarette smoking, nicotine dependence and anxiety disorders: a systematic review of populationbased, epidemiological studies. BMC Medicine 2012 10:123.

\section{Submit your next manuscript to BioMed Central and take full advantage of:}

- Convenient online submission

- Thorough peer review

- No space constraints or color figure charges

- Immediate publication on acceptance

- Inclusion in PubMed, CAS, Scopus and Google Scholar

- Research which is freely available for redistribution

Submit your manuscript at www.biomedcentral.com/submit
C Biomed Central 